Fortgeschrittenes NSCLC

\title{
Längeres Überleben durch Erhaltungstherapie
}

\section{Nach erfolgreicher Firstline-Therapie kann eine kontinuierliche Pemetrexed- Gabe die progressionsfreie Zeit sowie das Gesamtüberleben verlängern.}

In der PARAMOUNT-Studie hatten 539 Patienten mit einem fortgeschrittenen oder metastasierten nicht-kleinzelligen Lungenkarzinom (NSCLC) ohne Plattenepithelhistologie auf die Induktionstherapie mit Pemetrexed (Alimta ${ }^{\circledast}$ ) und Cisplatin gut angesprochen. Die Studienteilnehmer wurden im Verhältnis 2:1 randomisiert mit Pemetrexed oder Placebo bis zum Progress weiterbehandelt. Unter der Erhaltungstherapie lag das progressionsfreie Überleben bei 4,1 Monaten gegenüber 2,8 Monaten unter Placebo [Paz-Ares L et al.
Lancet Oncol. 2012;13(3):247-55]. Auch beim Gesamtüberleben bot die Erhaltungstherapie signifikante Vorteile, so Martin Kohlhäufl, Stuttgart: Ab Beginn der Induktionstherapie betrug die mediane Überlebenszeit in der Verumgruppe 16,9 Monate gegenüber 14,0 Monaten im Placebo-Arm. Das Mortalitätsrisiko wurde durch die kontinuierliche PemetrexedErhaltungstherapie im Vergleich zu Placebo um $22 \%$ reduziert. Von diesem Überlebensvorteil profitierten in der Studie alle relevanten Subgruppen. Einer Umfrage zufolge würde die Mehrzahl der Betroffenen einer Erhaltungstherapie zustimmen [Peeters L. J Thorac Oncol. 2012;7(8):129195]. Auch bei einem Lebenszeitgewinn von nur einem Monat würden sich knapp $50 \%$ der Patienten für eine Erhaltungstherapie entscheiden. Bei einer zu erwartenden Überlebensverlängerung von einem Jahr sprachen sich nahezu alle Patienten für eine Erhaltungstherapie aus. Abdol Ameri

Mediadinner "NSCLC - Ein Jahr Erhaltungstherapie mit Pemetrexed" anlässlich der Jahrestagung der Deutschen, Österreichischen und Schweizerischen Gesellschaften für Hämatologie und Onkologie, Stuttgart, 19. Oktober 2012; Veranstalter: Lilly

\section{Neue Optionen erhöhen Therapiechancen}

Neue Substanzen haben das Überleben von Myelom-Patienten erheblich verlängert. Auf die Stammzelltransplantation sollte man wenn möglich aber nicht verzichten.

Mit Einführung der Hochdosis-Chemotherapie und anschließender autologer Stammzelltransplantation (ASZT) hat sich die Prognose des multiplen Myeloms verbessert. Auch die Integration von Substanzen wie Lenalidomid (Revlimid ${ }^{\circ}$ ) in die Therapie trug dazu bei. Hermann Einsele, Würzburg, verwies auf die Studie MM-020, in der die frühere Standardtherapie Melphalan/Prednison (MP) dem neuen MPR-Tripleregime (MP + Lenalidomid) mit folgender Lenalidomid-Erhaltungstherapie klar unterlegen war: Das progressionsfreie Überleben unter der Addition von Lenalidomid fiel erheblich länger aus als im Kontrollarm (31 vs. $12 \mathrm{Mo}-$ nate). Unter Lenalidomid traten jedoch häufiger Zweitkarzinome auf. Nach Einsele war das Risiko für Zweittumoren aber geringer als für Progress oder Tod und die Therapie-Intensivierung damit eindeutig von Nutzen. Bislang führt die Stammzelltransplantation zu besseren Ergebnissen als die rein medikamentöse Behandlung. In einer Phase-III-Studie erhielten Patien- ten nach initialer Therapie mit Lenalidomid/Dexamethason randomisiert das MPR-Regime oder eine Tandem-ASZT, jeweils gefolgt von der Lenalidomid-Erhaltungstherapie. Das Ansprechen war in beiden Gruppen vergleichbar, das progressi- onsfreie 2-Jahres-Überleben lag jedoch im Transplantationsarm mit 73 vs. $54 \%$ deutlich höher [Palumbo A et al. ASH 2011; Abstr. 3069].

Katharina Arnheim

\section{Satellitensymposium „Neues und Bewährtes} beim Multiplen Myelom und MDS" anlässlich der Jahrestagung der Deutschen, Österreichischen und Schweizerischen Gesellschaften für Hämatologie und Onkologie, Stuttgart, 20. Oktober 2012; Veranstalter: Celgene

Fortgeschrittenes Mammakarzinom

\section{Studie bestätigt Therapieerfolg durch mTOR-Inhibition}

\section{Patientinnen mit Hormonrezeptor- positivem und HER2-negativem \\ Mammakarzinom in fortgeschrittenen Stadien profitieren von der Behand- lung mit einem mTOR-Inhibitor.}

Mit Everolimus (Afinitor ${ }^{\circledR}$ ) steht der erste und einzige Inhibitor des mammalian-Target-Of-Rapamycin (mTOR)-Signalwegs zur Verfügung, der für postmenopausale Patientinnen mit fortgeschrittenem Hormonrezeptor-positiven, HER2-negativen Mammakarzinom zugelassen ist.

Die Ergebnisse der Phase-III-Studie BOLERO-2 mit 724 Patientinnen zeigen ein medianes progressionsfreies Überleben von 11,0 Monaten unter Everolimus in Kombination mit Exemestan - verglichen mit nur 4,1 Monaten unter Placebo plus Exemestan [Piccart $\mathrm{M}$ et al. J Clin Oncol. 2012;30(Suppl.):Abstr. 559]. Die Analyse zum klinischen Nutzen (definiert als Summe aus komplettem und partiellem Ansprechen sowie stabiler Erkrankung über mindestens 24 Wochen) ergab, dass in der Everolimus-Gruppe $51,3 \%$ der Patientinnen profitierten, im Placebo-Arm waren dies nur 26,4\%.

Nach Informationen von Novartis Pharma 